\title{
Modelagem e simulação de dados hidrológicos na Bacia do Rio lbicuí da Armada
}

Modeling and simulation of hydrological data at Ibicuí da Armada River Basin

\author{
Thiago Boeno Patricio Luiz'
}

\begin{abstract}
RESUMO
O uso de modelos hidrológicos é amplamente utilizado para fins de conhecimento dos regimes de vazões em bacias hidrográficas e para dar suporte quando ocorrem falhas nas medições. O presente estudo teve por objetivo calibrar um modelo hidrológico na bacia hidrográfica do rio Ibicuí da Armada no Sudoeste do Rio Grande do Sul, a fim de simular seu regime de vazões diários para um período de 250 dias à frente. Utilizou-se o programa WIN_IPH2 para simulação, ajustando parâmetros de otimização através de testes de calibração automática por funções-objetivo e, verificando posteriormente o seu ajuste. As séries diárias foram adquiridas junto ao banco de dados disponibilizado pela Agência Nacional das Águas (ANA) e pelo Instituto Nacional de Meteorologia (INMET). A função-objetivo que apresentou o melhor ajuste amostral entre as séries foi a função de Desvio Médio Absoluto (DMA), mostrando-se o melhor para representar a Q90 e a Q95. O emprego do software WIN_IPH2 aliado a informações condicionantes dos regimes de vazões mostraram-se bastante úteis para utilização em situações que demandem o conhecimento de disponibilidade hídrica, contribuindo, assim, para a melhora das ações de gestão dos recursos hídricos.
\end{abstract}

Palavras-chave: Modelos hidrológicos; WIN_IPH2; Simulação de vazões

ABSTRACT

The use of hydrological models is widely used for information purposes of flow regimes in watersheds and to support when failures occur in measurements. This study aimed to calibrate a hydrologic model at Ibicuí da Armada watershed on southwest of Rio Grande do Sul State, in order to simulate their regime of daily flows for a period of 250 days ahead. Were used the WIN_IPH2 software for the simulation, adjusting optimization parameters through automatic calibration tests by objective functions, and subsequently checking it's fit. The daily series were acquired from the database provided by Agência Nacional de Águas (ANA) and the Instituto Nacional de Meteorologia (INMET). The objective function that showed the best fit between the sample series was Mean Absolute Deviation function (MAR), proving to be the best to represent the Q90 and Q95. The employment created by WIN_IPH2 software combined with information constraints of flow regimes were quite useful for use in situations that require knowledge of water availability, thus contributing to the improvement of the management actions of water resources.

Keywords: Hydrologic models; WIN_IPH2; Flow simulation

\section{INTRODUÇÃO}

O monitoramento de dados hidrológicos em uma bacia hidrográfica é fundamental para realização de estudos e projetos que envolvem recursos hídricos. A inexistência ou a falta de qualidade das informaçōes das bacias hidrográficas brasileiras é um dos principais problemas envolvendo os estudos hidrológicos, onde muitas vezes é preciso utilizar técnicas de regionalização dos dados disponíveis nas regiôes vizinhas, o que acaba baixando a confiabilidade das amostras e, consequentemente, gerando incertezas nos processos de simulação de cenários.

Outro fator limitante são os custos envolvidos na implantação e operação de redes hidrométricas em um país territorialmente tão grande quanto o Brasil. Nessa perspectiva, torna-se importante melhorar as informaçóes disponíveis, e o uso de modelos hidrológicos vem sendo de grande utilidade para diversas áreas de planejamento e de estudos técnicos, servindo de base para um gerenciamento mais adequado e sustentável das bacias hidrográficas. 
Segundo Bravo et al. (2007), a modelagem hidrológica utiliza métodos para estudar os complexos processos que englobam o ciclo hidrológico, sendo os sistemas que simulam o processo de transformação da chuva em vazão os mais utilizados. Já Tucci (2005) afirma que a modelagem é uma ferramenta que equaciona os processos hidrológicos, a fim de representar e entender o comportamento da bacia hidrográfica e prever condiçóes diferentes das observadas. Através da modelagem é possível antecipar eventos de modo a permitir que medidas preventivas sejam tomadas

Para este estudo utilizou-se o Modelo hidrológico para Grandes Bacias (MGB) do Instituto de Pesquisas Hidráulicas da Universidade Federal do Rio Grande do Sul (IPH/UFRGS) proposto inicialmente por Collischon (2001), utilizando o software WIN_IPH2 desenvolvido por Bravo et al. (2006). O modelo consiste em uma análise de uma série de dados e parâmetros físicos da bacia hidrográfica, objetivando a simulação hidrológica a partir de séries históricas de dados de vazão, precipitação e evapotranspiração visando a simulação de vazôes, através da obtenção de parâmetros de otimização para o modelo.

No programa WIN_IPH2 é utilizado o algoritmo SCE-UA (Shuffled Complex Evolution - University of Arizona) de Duan et al. (1994) para a calibração automática monobjetivo dos modelos. Este algoritmo possui características semelhantes a um algoritmo genético, utilizando-se de sucessivas iterações e avaliações da função-objetivo visando a convergência de valores de otimização do sistema.

O modelo MGB-IPH já foi utilizado anteriormente com sucesso em diversas avaliaçóes técnicas e em bacias de grande porte como a Bacia do Rio Uruguai (TUCCI et al., 2003) e a Bacia do Rio São Francisco (TUCCI et al., 2005).

\section{METODOLOGIA}

\section{I CaracterizaçÃo da ÁREA de ESTUdo}

A Bacia Hidrográfica do Rio Ibicuí da Armada está localizada entre os municípios de Rosário do Sul, Santana do Livramento e Dom Pedrito, na regiáo Sudoeste do Estado do Rio Grande do Sul, ilustrada na Figura 1. O rio Ibicuí da Armada é contribuinte do rio Santa Maria e faz parte da bacia hidrográfica Santa Maria U-070 (SEMA, 2005) sendo parte da região hidrográfica do rio Uruguai.

Segundo os dados da ANA, a bacia em estudo possui uma área de drenagem de aproximadamente $6010 \mathrm{~km}^{2}$

Figura 1 - Localização da bacia hidrográfica do rio Ibicuí da Armada

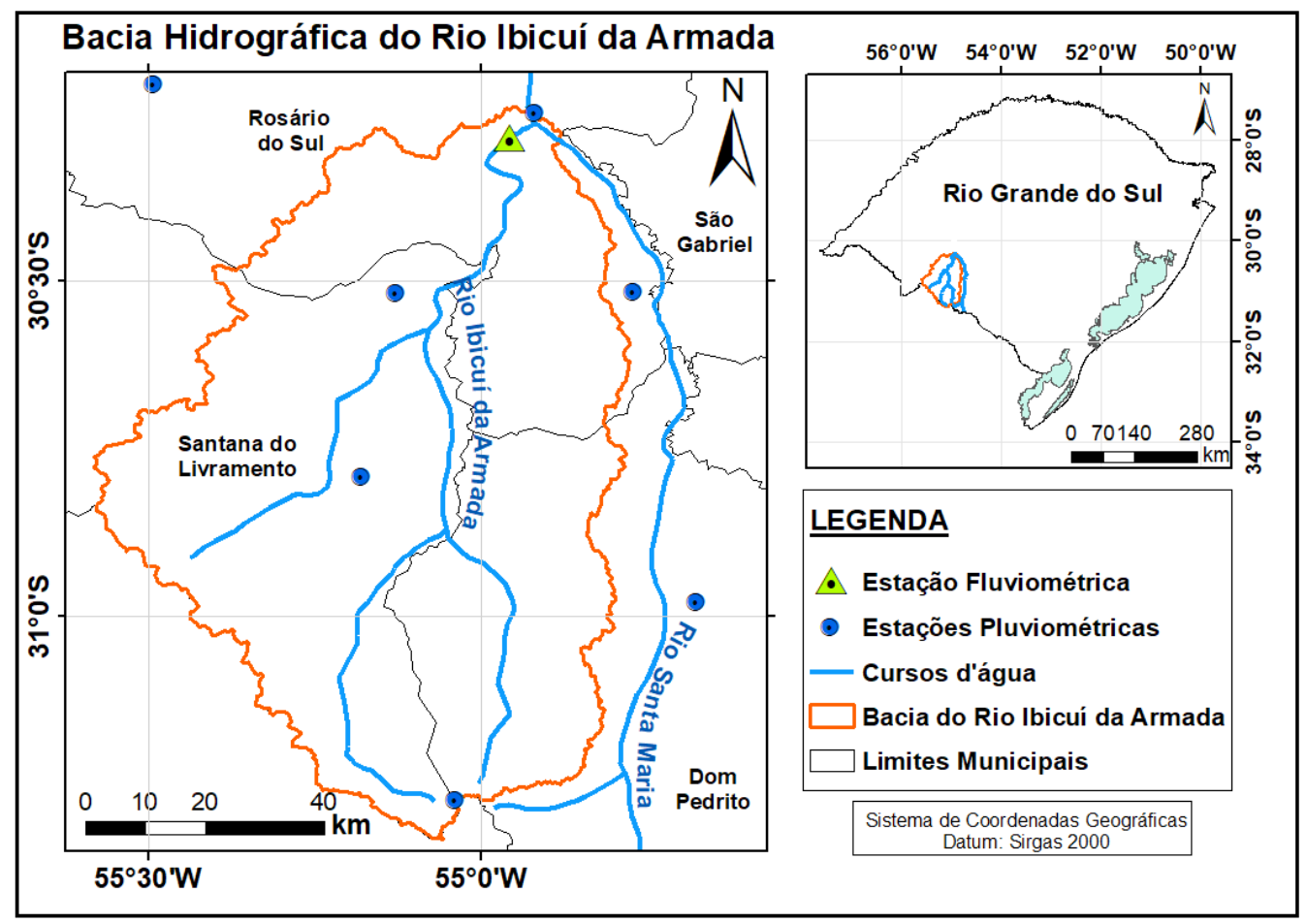

Fonte: SEMA (2005). 
e precipitaçóes médias anuais em torno de $1500 \mathrm{~mm}$. O clima em todo o Sudoeste do Rio Grande do Sul é definido segundo classificação de Köppen (ALVARES et al., 2013), como subtropical úmido, tipo CFa, caracterizado pela presença de invernos frios, veróes quentes e inexistência de estação seca. O uso e a ocupação do solo na bacia estão associados à prática da pecuária e agricultura segundo o Instituto Brasileiro de Geografia e Estatística (IBGE, 2010).

\subsection{LEVANTAMENTO DE DADOS E MODELO HIDROLÓGICO UTILIZADO}

As séries de dados de vazão e precipitação foram obtidos do Sistema de Informações Hidrológicas (HidroWeb) mantido pela ANA. Para a determinação da evapotranspiração, foi utilizado o método de Thornthwaite (THORNTHWAITE, 1948), que estima a evapotranspiração mensal através da temperatura utilizando um fator de correção de latitude. Esses dados foram adquiridos junto a estação meteorológica do Instituto Nacional de Meteorologia (INMET), da cidade de Bagé/RS. As estaçôes utilizadas no trabalho estão listadas na Tabela 1.

Tabela 1 - Estaçóes utilizadas

\begin{tabular}{|c|c|c|c|c|}
\hline Código & Nome & Tipo & Latitude & Longitude \\
\hline 76300000 & Ponte Ibicuí da Armada & Fluviométrica & S $30^{\circ} 16^{\prime} 50.88^{\prime \prime}$ & 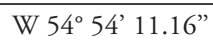 \\
\hline 03055007 & São Carlos & Pluviométrica & S $30^{\circ} 12^{\prime} 15.12^{\prime \prime}$ & W $55^{\circ} 29^{\prime} 30.84^{\prime \prime}$ \\
\hline 03054007 & Rosário do Sul & Pluviométrica & S $30^{\circ} 14^{\prime} 48.84^{\prime \prime}$ & W $54^{\circ} 55^{\prime} 3.00^{\prime \prime}$ \\
\hline 03055005 & Santa Rita & Pluviométrica & S $30^{\circ} 30^{\prime} 59.04^{\prime \prime}$ & W $55^{\circ} 7^{\prime} 36.12^{\prime \prime}$ \\
\hline 03054016 & Granja Umbu & Pluviométrica & S $30^{\circ} 30^{\prime} 51.84^{\prime \prime}$ & W $54^{\circ} 46^{\prime} 13.08^{\prime \prime}$ \\
\hline 03055009 & Livramento BR-293 & Pluviométrica & S $30^{\circ} 47^{\prime} 30.12^{\prime \prime}$ & W $55^{\circ} 10^{\prime} 45.12^{\prime \prime}$ \\
\hline 03054002 & Dom Pedrito & Pluviométrica & S $30^{\circ} 58^{\prime} 41.16^{\prime \prime}$ & W $54^{\circ} 40^{\prime} 32.88^{\prime \prime}$ \\
\hline 03155001 & Três Vendas & Pluviométrica & $S 31^{\circ} 16^{\prime} 31.08^{\prime \prime}$ & W $55^{\circ} 2^{\prime} 13.92^{\prime \prime}$ \\
\hline 83980 & Bagé & Meteorológica & S $31^{\circ} 20^{\prime} 52.08^{\prime \prime}$ & W 54 0’ 47.85” \\
\hline
\end{tabular}

Para a simulação das vazóes na bacia, utilizou-se o software WIN_IPH2 (BRAVO et al. 2006). Inicialmente, realizou-se a calibração, que é a técnica numérica para estimar valores para o modelo, buscando a melhor otimização para as variáveis e permitindo obter valores simulados próximos aos valores observados. As funcionalidades do aplicativo permitem a calibração manual e a calibração automática, sendo os resultados apresentados em texto e em gráficos.

O ajuste dos valores dos parâmetros para este estudo foi realizado através de calibração automática monobjetivo utilizando diferentes funçôes-objetivo, que são as equações utilizadas para calcular uma medida numérica da diferença entre os resultados do modelo e os valores observados. Foram testadas as seguintes funçóes objetivo: Coeficiente Kling-Gupta, Desvio Médio Absoluto, Desvio Quadrado Relativo Médio, Desvio Quadrado Inverso, Coeficiente de Nash-Sutcliffe, Desvio Relativo Médio e Erro de Volume.

Para efetuar a simulação, selecionaram-se intervalos de tempo sem falhas nas medições. O período escolhido para a calibração foi de 07 de março de 2000 a 30 de abril de 2003, totalizando 1150 dias, onde a série não apresentou precipitação nos 10 primeiros dias. Para a verificação do modelo, utilizou-se o período de 29 de julho de 2003 a 05 de abril de 2004, totalizando 250 dias.

\subsection{CÁlCULO DOS DADOS HIDROLÓGICOS DA BACIA}

Tendo em vista que as precipitaçóes não são uniformes no espaço, e, de modo a obter uma estimativa de chuva que representasse a área total da bacia, utilizou-se o método dos polígonos de Thiessen entre 7 estaçóes pluviométricas existentes na área usando o software ArcGIS 10.2.2 (ESRI, 2014). Dessa forma, foi definida uma representatividade para cada estação pluviométrica, baseadas em suas localizaçóes geográficas na bacia, gerando uma única série de precipitação mais adequada para a calibraçáo dos dados. Assim, as precipitaçóes de cada estação foram multiplicadas por seu valor de abrangência, gerando uma precipitação mais representativa da área total da bacia hidrográfica.

O tempo de concentração (Tc) foi determinado através da Equação 1 de Dodge (PORTO, 1995), indicada para bacias de maior porte, utilizando dados extraídos do modelo digital de elevação.

Onde:

Tc $=$ minutos

$\mathrm{Tc}=21,88 \mathrm{~A}^{0,41} \mathrm{~S}_{\circ}{ }^{-0,17}$

Área $(\mathrm{A})=\mathrm{km}^{2}$

Declividade $\left(\mathrm{S}_{\mathrm{o}}\right)=\mathrm{m} / \mathrm{m}$ 


\section{RESULTADOS E DISCUSSÕES}

Como dado de entrada, o tempo de concentração estimado para a Bacia Hidrográfica do Rio Ibicuí da Armada utilizando a Equaçáo 1 foi de aproximadamente 48 horas. Outro dado de entrada para realizar a calibração do modelo é a informação da área impermeável da bacia. Esse dado foi estimado a partir da soma das áreas urbanas de cada município pertencentes à bacia, utilizando, para tal, imagens do satélite Landsat 5 Thematic Mapper (USGS, 2015). Dessa forma, aproximou-se o valor de impermeabilização em 8 \% da área total, baseado na construção de um arquivo do tipo shapefile sobre as áreas urbanas pertencentes a bacia hidrográfica e fazendo posteriormente sua soma $\mathrm{em}^{2}$.

\section{I Calibração}

Durante a calibração, ajustaram-se 500 geraçôes de repetiçôes, testando a função objetivo que apresentasse o menor erro possível entre a série observada e a ajustada. A função objetivo que apresentou melhores resultados foi a de Desvio Médio Absoluto. Os valores ajustados e observados apresentaram uma boa correlação, indicando que o modelo se ajustou para a série de dados (Figura 2).

Figura 2 - Gráfico da etapa de calibração utilizando a função-objetivo Desvio Médio Absoluto.

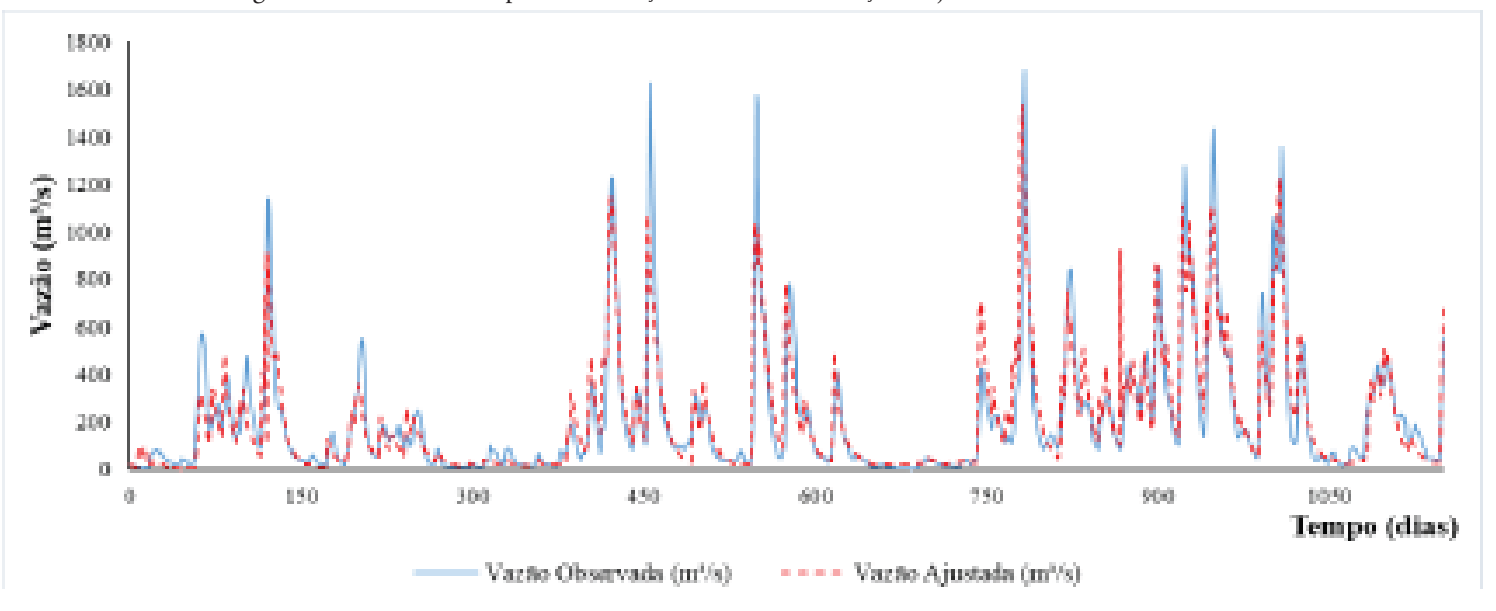

Fonte: Autor

Entre a série observada e a ajustada, observa-se que o modelo apresenta alguns pontos onde não conseguiu um bom ajuste, principalmente entre alguns picos de vazão maiores que $1000 \mathrm{~m}^{3} / \mathrm{s}$. No entanto, o modelo mostra-se satisfatório no ajuste para vazóes de menor volume. Esse fato é evidenciado ao plotar-se as curvas de permanência das séries ajustadas e observadas (Figura 3).

Figura 3 - Curvas de permanência em escala logarítmica da série observada e ajustada.

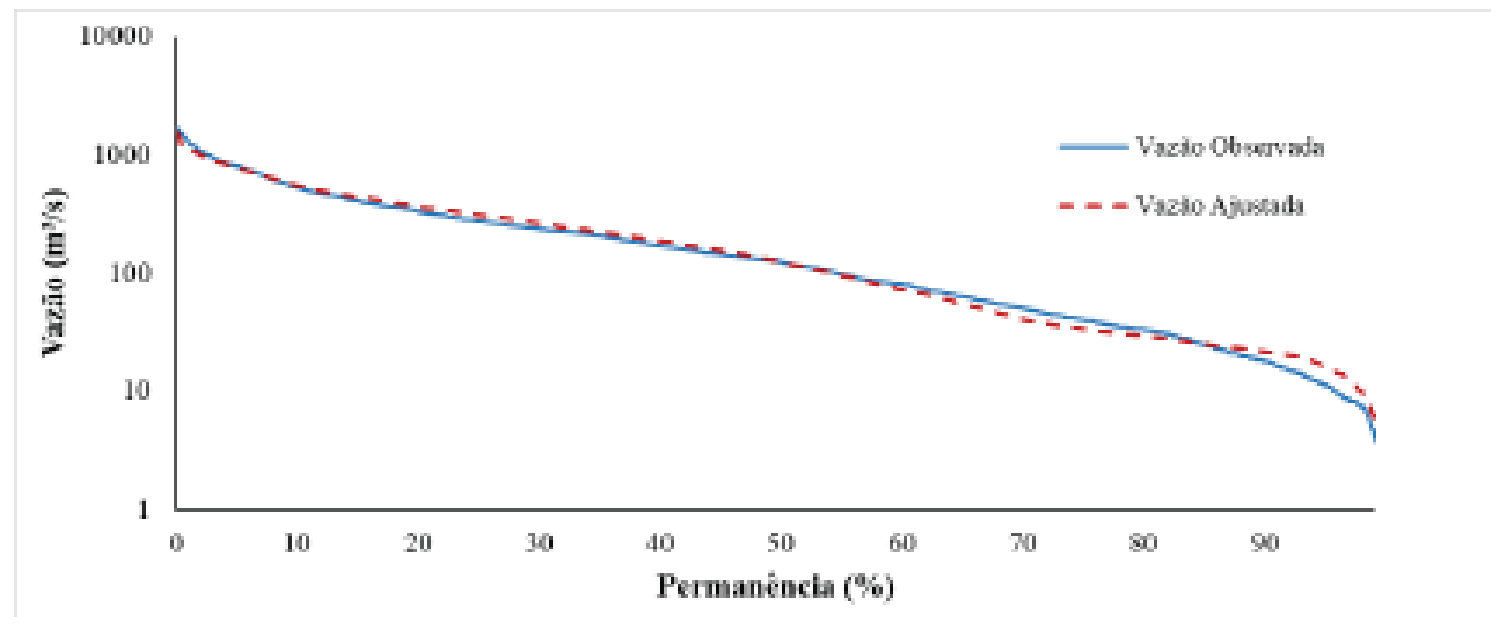

Fonte: Autor 
Ao observar as curvas de permanência das séries observada e ajustada, nota-se que as vazóes possuem boa convergência, o que configura um bom ajuste. Contudo, o modelo apresenta uma pequena alteração entre as curvas, mostrando valores preditos um pouco abaixo do real entre a $\mathrm{Q}_{60}$ e $\mathrm{Q}_{80}$, e um pouco acima entre a $\mathrm{Q}_{85}$ e a $\mathrm{Q}_{99}$.

Modelos criados a partir de dados observados visam sempre uma representação teórica de um fenômeno real a partir de um método abstrato. Nessa representação, sempre haverá algum erro, devendo sempre analisá-lo antes de sua aplicação. Diante disso, a fim de avaliar os erros cometidos, elaborou-se um gráfico de resíduos (figura 4) bem como sua linha de tendência para a verificação de seu ajuste.

Ao observar o gráfico da figura 4, embora ocorra alguns outliers, verifica-se uma boa correlação dos dados observados e ajustados da série, evidenciado pelo coeficiente de determinação $R^{2}=0,79$. A medida de ajustamento do modelo criado em relação aos valores observados indica um ajuste de $79 \%$.

Figura 4 - Gráfico dos resíduos do modelo.

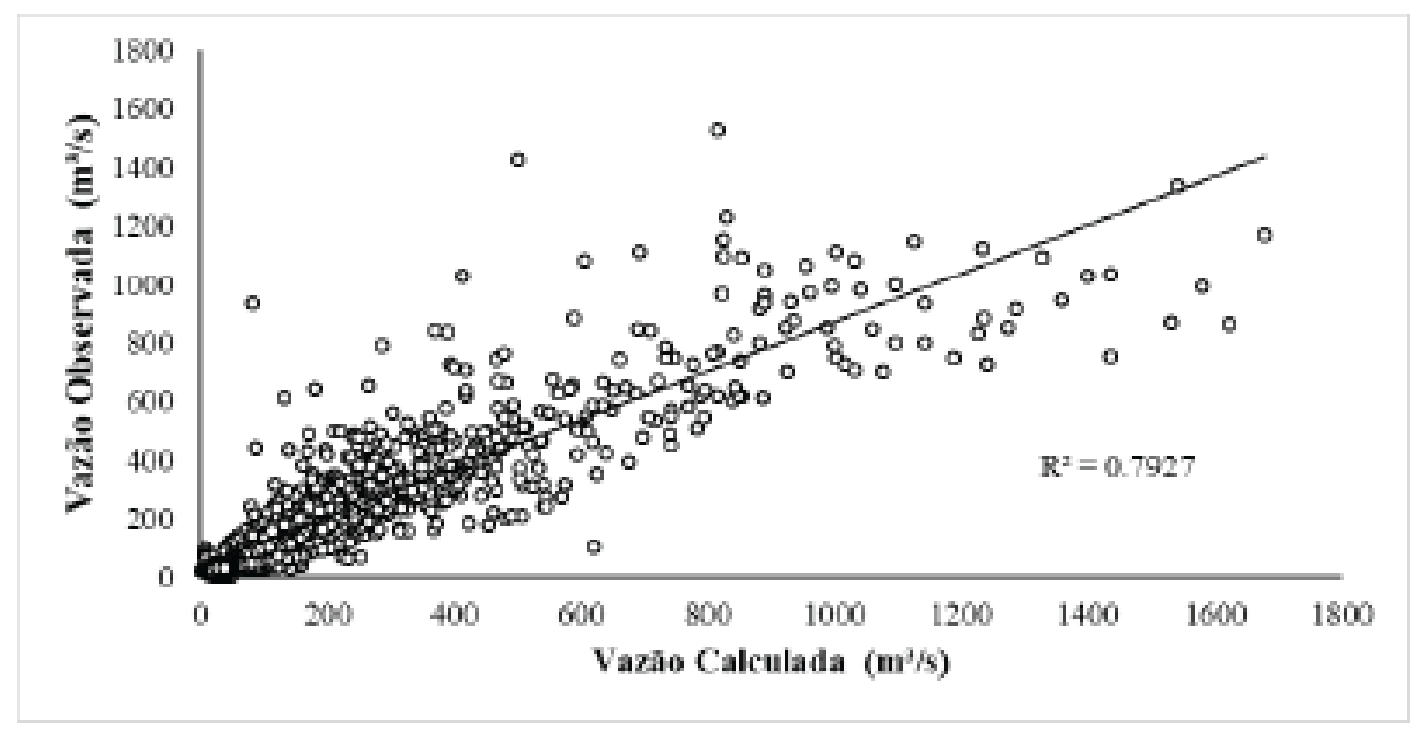

Fonte: Autor

\subsection{VERIFICAÇÃo}

Para a verificação do modelo, selecionou-se um período de 250 dias posteriores a calibração. O procedimento consiste em verificar a validade do modelo construído através da comparaçáo com a série real observada ao longo do tempo posterior ao utilizado na modelagem. (Figura 5).

Figura 5 - Gráfico da etapa de verificação do modelo.

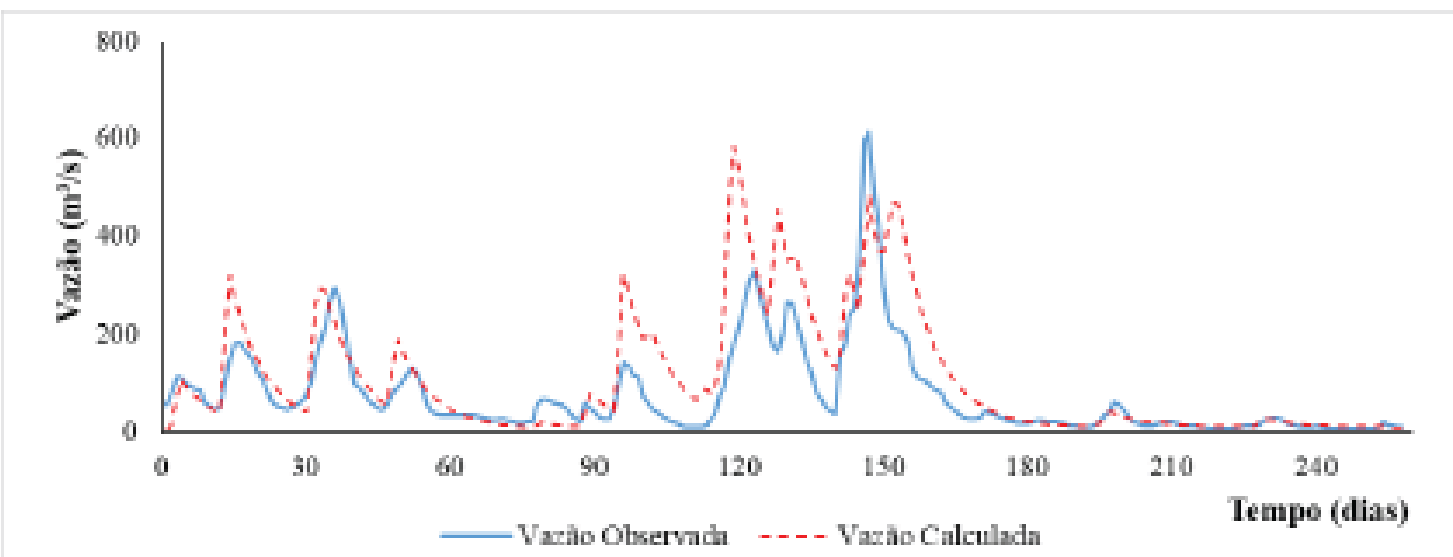

Fonte: Autor 
Ao observar as estimativas das vazóes calculadas e das observadas, evidencia-se boas correlaçóes entre as séries, onde a série calculada apresentou tendências de crescimento e de decaimento semelhante a série observada. Dessa forma, tendo em vista os resultados positivos da etapa de análise dos resíduos e da etapa de verificação do modelo, avaliou-se que o programa WIN_IPH2 apresentou bons resultados para simular as vazóes na bacia hidrográfica do rio Ibicuí da Armada.

\section{CONCLUSÕES}

A utilização do programa WIN_IPH2 na representação do comportamento hidrológico de bacias, aliada a informaçóes referentes ao comportamento hidrodinâmico no sistema permite compreender com maior facilidade os fenômenos envolvidos nos modelos chuva-vazão.

A simulação dos dados de vazão, obtidas a partir das séries de vazão, precipitação e evapotranspiração observada apresentaram boa margem de acerto, evidenciado pelo gráfico de resíduos, configurando-se um modelo hidrológico interessante para realizar estimativas de vazões em grandes bacias hidrográficas.

Dessa forma, o modelo apresentou-se satisfatório para realizar a simulaçáo de dados hidrológicos na bacia hidrográfica do rio Ibicuí da Armada, configurando uma ferramenta para subsidiar o gerenciamento dos recursos hídricos da região.

\section{AGRADECIMENTOS}

Em especial à Universidade Federal de Santa Maria pela estrutura e apoio fornecido para o desenvolvimento do trabalho e à Coordenação de Aperfeiçoamento de Pessoal de Nível Superior pela bolsa de pós-graduação concedida ao autor.

\section{REFERÊNCIAS}

ALVARES, C.A.; STAPE, J.L.; SENTELHAS, P.C.; GONÇALVES, J.L.M.; SPAROVEK, G. Köppen's climate classification map for Brazil. Meteorologische Zeitschrift, v. 22, n. 6, p. 711-728, 2013.

BRASIL. Instituto Brasileiro de Geografia e Estatística - IBGE. Disponível em: <www.ibge.gov.br>. Acesso em: 10 out. 2017.

BRASIL. Instituto Nacional de Metereologia - INMET. BdMep. Disponível em: <http://www.inmet.gov.br/portal/>. Acesso em: 13 de dez. 2017.

BRASIL. Ministério do Meio Ambiente. Agência Nacional de Águas - ANA. Sistema de informaçôes hidrológicas HidroWeb. Disponível em: <http://hidroweb.ana.gov.br/>. Acesso em: 13 de nov. 2017.

BRAVO, J. M.; ALLASIA, D.; TASSI, R.; MELLER, A.; COLLISCHON, W.; TUCCI, C. E. M. (2006). Manual de usuário do WIN_IPH2. Versão 1.0. Porto Alegre: IPH.

BRAVO, J. M.; ALLASIA, D. G.; COLLISCHONN, W.; TASSI, R.; MELLER, A.; TUCCI, C. E. M. (2007). Avaliação visual e numérica da calibração do modelo hidrológico IPH II com fins educacionais. In: Anais do XVII Simpósio Brasileiro de Recursos Hídricos e $8^{\circ}$ Simpósio de Hidráulica e Recursos Hídricos dos Países de Língua Portuguesa, São Paulo.

COLLISCHONN, W.; TUCCI, C. E. M. Simulação hidrológica de grandes bacias. Revista Brasileira de Recursos Hídricos. v. 6, n. 1, p.95-118, 2001.

DUAN, Q.; SOROOSHIAN, S.; GUPTA, V. - Optimal use of the SCE - UA global optimization method for calibrating watershed models. Journal of Hydrology, v. 158, n. 3-4, p. 265-284, 1994. 
ESRI. Environmental Systems Research Institute. ArcGIS for Desktop. Estados Unidos da América. Versão 10.2.2, 2014.

ESTADO DO RIO GRANDE DO SUL. SEMA. Secretaria Estadual de Meio Ambiente - Bacias hidrográficas e municípios do Rio Grande do Sul. Mapa. 2005.

PORTO, R. Escoamento Superficial Direto. In: Drenagem Urbana. Org. por Tucci, C. E. M.; Porto, R. De; Barros, M. T. ABRH, ed. UFRGS, Porto Alegre - RS. 1995.

THORNTHWAITE, C. W. An approach toward a rational classification of climate. Geographical Review, New York, 1948.

TUCCI, C.E.M.; CLARKE, R.T.; COLLISCHONN W.; DIAS, P.L.S.; SAMPAIO, G.O. Long term flow forecast based on climate and hydrological modeling: Uruguay river basin. Water Resources Research. v. 39, n. 7, 2003.

TUCCI, C. E. M. Modelos hidrológicos. 2 ed. Porto Alegre: UFRGS/ABRH. 678 p. 2005.

TUCCI, C.E.M.; SILVA, B.C.; COLLISCHONN W.; MARENGO, J. A.; SAMPAIO, G.O.; CHOU S.C.; DIAS, P.L.S. Previsão de Vazão de Longo Prazo na Bacia do Rio São Francisco III: Previsão da Vazão. Revista Brasileira de Recursos Hídricos. 13 p. 2005.

USGS. UNITED STATES GEOLOGICAL SURVEY. Landsat-5 (TM). Disponível em: http://landsat.usgs.gov. Acesso em: 23 mai. 2015. 